\title{
Tumor heterogeneity in muscle-invasive bladder cancer
}

\author{
Ho Won Kang ${ }^{1,2}$, Wun-Jae Kim ${ }^{1,2}$, Woonyoung Choi ${ }^{3}$, Seok Joong Yun ${ }^{1,2}$ \\ ${ }^{1}$ Department of Urology, School of Medicine and Medical Research Institute, Chungbuk National University, Cheongju, Korea; ${ }^{2}$ Department of \\ Urology, Chungbuk National University Hospital, Cheongju, Korea; ${ }^{3}$ Johns Hopkins Greenberg Bladder Cancer Institute and Brady Urological \\ Institute, Johns Hopkins University, Baltimore, MD, USA \\ Contributions: (I) Conception and design: All authors; (II) Administrative support: None; (III) Provision of study materials or patients: None; (IV) \\ Collection and assembly of data: None; (V) Data analysis and interpretation: All authors; (VI) Manuscript writing: All authors; (VII) Final approval of \\ manuscript: All authors. \\ Correspondence to: Seok-Joong Yun, MD, PhD. Department of Urology, Chungbuk National University, College of Medicine and Institute for Tumor \\ Research, 1st Chungdae-ro, Seowon-gu, Cheongju, Chungbuk 28644, Korea. Email: sjyun@chungbuk.ac.kr.
}

\begin{abstract}
Muscle-invasive bladder cancer (MIBC), a highly heterogeneous disease, shows genomic instability and a high mutation rate. Clinical outcomes are variable and responses to conventional chemotherapy differ among patients (due to inter-patient tumor heterogeneity and inter-tumor heterogeneity) and even within each individual tumor (intra-tumor heterogeneity). Emerging evidence indicates that tumor heterogeneity may play an important role in cancer progression, resistance to therapy, and metastasis. Comprehensive molecular subtyping classifies MIBC into distinct categories that have potential to guide prognosis, patient stratification, and treatment. Genomic characterization of timeseries analyses at the single cell level, and of cell-free circulating tumor DNA or circulating tumor cells, are emerging technologies that enable dissection of the complex clonal architecture of MIBC. This review provides insight into the clinical significance of the molecular mechanisms underlying heterogeneity, focusing on inter- and intra-tumor heterogeneity, with special emphasis on molecular classification and methods used to analyze the complex patterns involved.
\end{abstract}

Keywords: Tumor heterogeneity; bladder cancer (BC); biomarkers; tumor microenvironment (TME); molecular subtypes

Submitted Nov 20, 2019. Accepted for publication Feb 27, 2020.

doi: $10.21037 /$ tau.2020.03.13

View this article at: http://dx.doi.org/10.21037/tau.2020.03.13

\section{Introduction}

Urothelial carcinoma (UCC) of the urinary bladder is the most common form of bladder cancer (BC). It is highly heterogeneous at both the histologic and molecular levels (1). Although non-invasive, welldifferentiated tumors are relatively indolent, T1 high grade $\mathrm{BC}$ and muscle-invasive BC (MIBC) are a threat to life (2). MIBC is the most aggressive form of BC: 5 -year survival rates for patients with localized disease are $60 \%$, whereas those for patients with distant metastases are less than $10 \%$ (3). Systemic cisplatin-based combination chemotherapy is the first-line treatment for patients with metastatic BC; however, it is effective in only $30-40 \%$ of cases, and it is impossible to prospectively identify patients that will benefit (4). Neoadjuvant chemotherapy (NAC) followed by radical cystectomy is the standard treatment for MIBC; however, only about one third (25-50\%) of patients achieve a pathologic response (5). Thus, the need to identify markers that predict response to neoadjuvant cisplatinbased chemotherapy is urgent because patients who do not respond to NAC are at risk of unnecessary drug-related complications and delayed surgery $(6,7)$. Recently, immune checkpoint inhibitors atezolizumab, durvalumab, avelumab (targeting PD-L1), nivolumab, and pembrolizumab (targeting PD-1), along with the FGFR-targeted therapy erdafitinib, have been approved by the FDA for the treatment of advanced BC; however, unavailability of solid 


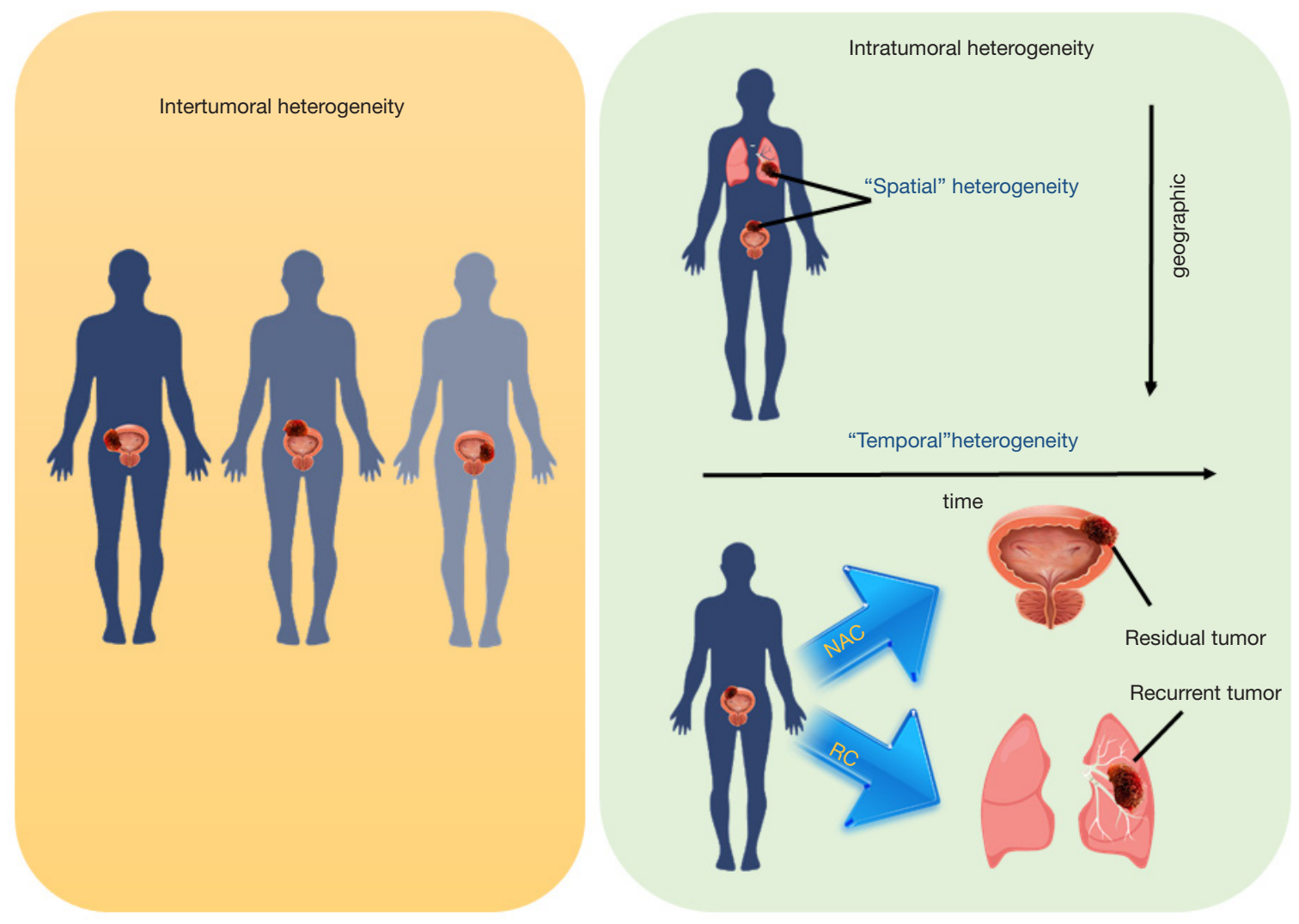

Figure 1 Scheme of tumor heterogeneity. Morphologic and functional differences between different patients with MIBC (inter-tumor heterogeneity, left panel), geographic differences between lesions within a single patient (spatial intra-tumor heterogeneity, right upper panel), and differences in the course of disease progression (temporal intra-tumor heterogeneity, left lower panel). Importantly, intra-tumor (spatio-temporal) heterogeneity leads to sampling bias and biomarker failure. NAC, neoadjuvant chemotherapy; RC, radical cystectomy.

tumor-specific biomarkers that predict treatment response limits the utility of these new therapeutic strategies in clinical practice $(8,9)$.

The design of novel and personalized therapies relies on a thorough understanding of MIBC biology. Recent therapeutic advances have been driven by identification of variations in tumor genotypes between patients, known as inter-patient heterogeneity, which predict responses to targeted treatments (10). Non-genetic phenotypic and functional heterogeneity are well recognized, as is heterogeneity of the tumor microenvironment (TME) (11). Comprehensive characterization of multiple tumor specimens obtained from the same patient suggests that marked intra-tumor heterogeneity might exist between geographical regions within the same tumor (spatial heterogeneity), as well as between the primary tumor and subsequent local or distant recurrences in the same patient (temporal heterogeneity) (Figure 1) (10,12). Therefore, inter- and intra-tumor heterogeneity are a hurdle in the path to personalized cancer medicine; this is because a single needle biopsy or surgical excision is unlikely to provide a clear picture of the genomic landscape of a patient's cancer (10). Sequencing technologies can characterize intratumor heterogeneity at the time of diagnosis, monitor clonal dynamics during treatment, and identify clinical resistance during disease progression $(10,13)$. The present review will provide a brief overview of the underlying mechanisms of tumor heterogeneity and their biological and clinical impact in MIBC. We will focus on new methods such as liquid biopsy and single cell RNA-seq (scRNA-seq) analysis, and explain how these techniques can help to decipher tumor heterogeneity in MIBC. 

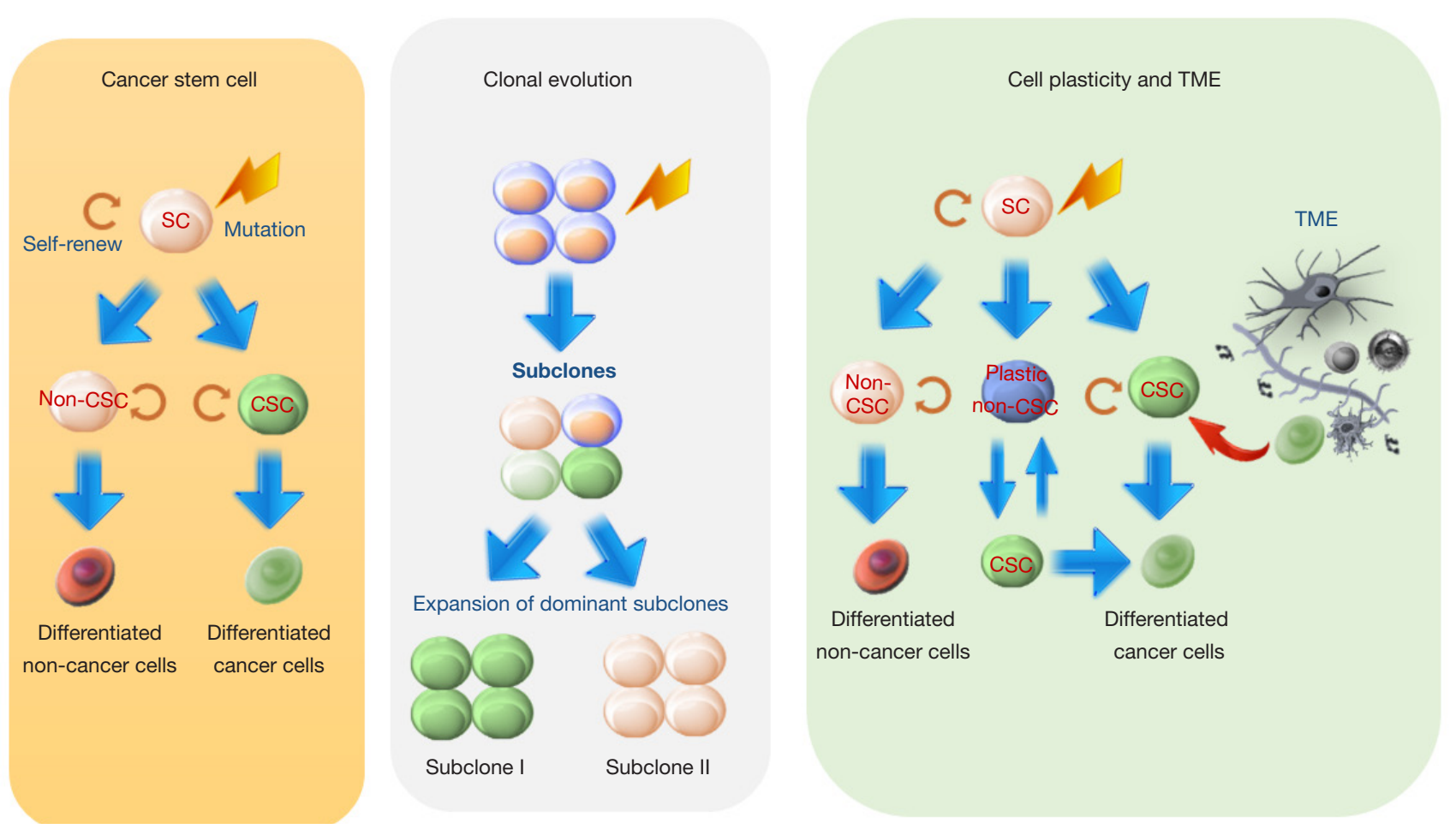

Figure 2 Scheme of tumor heterogeneity model. In the CSC (hierarchy) model, only CSCs generate tumors. In this model, CSC-tonon-CSC conversion is a unidirectional process (left panel). In the clonal evolution (stochastic) model, every tumor cell can stochastically generate a tumor (middle panel). The third model, the cancer cell plasticity model, posits bidirectional conversion between CSCs and nonCSCs in response to a changing TME. SC, stem cell; CSC, cancer stem cell; TME, tumor microenvironment.

\section{Mechanisms underlying tumor heterogeneity}

Cancer heterogeneity arises through introduction of genetic and epigenetic alterations due to genomic instability (14). Currently, there are two theories that describe development and maintenance of tumors: clonal evolution (the stochastic model) and the cancer stem cell (CSC) hypothesis (the hierarchical model) (Figure 2) $(15,16)$. Although these models are different (they place differing weight on the importance of stem cells and the microenvironment), they are not mutually exclusive (15).

The clonal evolutionary model of cancer development proposed by Nowell builds upon Darwinian models of natural selection: genetically unstable cells accumulate genetic mutations; selective pressure then favors growth and survival of subpopulations that harbor an advantage in terms of biological fitness $(12,17)$. Genetic instability within the tumor cell population leads single cells to accumulate additional mutations (18). This establishes genetically divergent clonal subpopulations; the most aggressive cells within these populations drive disease progression (19). This dynamic process leads to continuous tumor remodeling, with distinct dimensions of heterogeneity (13).

The CSCs hypothesis suggests that only a subset of cancer cells participate in "clonal" evolution and drive tumor progression; other cells are "evolutionary dead ends" (13). CSCs are interesting therapeutic targets due to their supposed role in tumorigenesis, metastasis, and chemoresistance $(20,21)$. Chemoresistance seems to be closely related to many intrinsic or acquired properties of CSCs, including quiescence, specific morphology, the ability to repair DNA, overexpression of anti-apoptotic proteins, the ability to pump out drugs (conferred by several ATP-binding cassette transporters), and production of detoxifying enzymes (22). The specific microenvironment (niche) and stability under hypoxic conditions provide CSCs with additional protection against anticancer therapies $(22,23)$. Studies of the phenotypic and functional properties of urothelial CSCs show that these cells cannot be characterized by a one-marker-fits-all approach; rather, markers such as CD44, CD44v6, cytokeratin 5 
(Krt5), CD47, the 67L-kDa laminin receptor $(67 \mathrm{LR})$, carcinoembryonic antigen-related cell adhesion molecule 6 (CEACAM6), and SOX4, along with high aldehyde dehydrogenase activity (ALDHhi), are used to isolate CSCs from patient specimens and to establish cancer cell lines successfully (24). Although many CSC markers are valuable for identification, isolation, and characterization of urothelial CSCs, the lack of consensus regarding these markers is the current major challenge in this field.

An alternative model, called reversible epithelial plasticity, provides a unifying framework that links the clonal evolution and CSC models together by postulating that cancer cells can interconvert between stem cell and differentiated states $(15,25)$. According to the plasticity model, processes intrinsic to tumor cells and/or various stimuli within the TME drive differentiated tumor cells to reacquire stem cell characteristics $(15,26)$. Conversely, these processes could also drive CSC differentiation toward nonstem cell characteristics (Figure 2) (15). In general, cancer cells display higher intrinsic or spontaneous plasticity than normal cells; studies have linked plasticity and stemness to regulation of epithelial-mesenchymal transition (EMT) $(15,27,28)$.

The interaction between tumor cells and the microenvironment is a key factor that regulates cancer development, progression, and metastasis (29). The TME is a dynamic network that comprises cancer cells, stromal tissue (immune cells, fibroblasts, myofibroblasts, cytokines, and vascular tissue), and the surrounding extracellular matrix (ECM) (30). Studies show that the TME not only plays a role in activating EMT in tumor cells, but also interacts with CSCs (Figure 2) (2). These neighboring cells trigger molecular signaling pathways that regulate CSC maintenance and survival; however, these pathways also induce drug resistance (31). In addition, tumor niche components such as the ECM shelter CSCs from therapeutic agents (31). Taken together, the interplay between CSC/EMT programs and the TME provides an opportunity to examine the nature of intratumor heterogeneity and offers insight into mechanisms underlying resistance to anticancer drugs (32).

\section{Inter-tumor heterogeneity in MIBC}

\section{Molecular markers of MIBC}

The clinical outcomes and responses of MIBCs to conventional treatment vary widely. Selecting the appropriate treatment depends on clinico-pathologic characteristics; however, current staging systems are very inaccurate, resulting in unacceptably high rates of clinical under-staging and, ultimately, to inadequate treatment $(33,34)$. Thus, efforts have been made to complement pathologic evaluation with objective molecular biomarkers (35). Detection of molecular markers in urine, tissue, or blood gives us the opportunity to increase our understanding of the biology of specific cancer and its micro- and macro-environment $(36,37)$. To date, studies have examined biomarkers associated with pathways playing important roles in tumor growth and progression; these include markers of cell cycle regulation, angiogenesis, apoptosis, and DNA damage repair (DDR) $(36,38,39)$. Several retrospective studies report that accumulation of p53 in cell nuclei is prognostic for MIBC, particularly in patients treated with radical cystectomy $(36,40)$. Indeed, tumors harboring TP53 mutations respond better to chemotherapy than those that do not (41). However, TP53 is not a clinically established prognostic biomarker for MIBC, despite over 100 studies evaluating its utility (36). A phase III trial that randomized patients after radical cystectomy for pT1-2N0M0 UCC into surveillance or adjuvant MVAC chemotherapy groups according to TP53 status did not confirm the prognostic value of $\mathrm{p} 53$; neither did it show that MVAC chemotherapy had any benefit for patients with p53-positive tumors (42). Anti-apoptotic biomarkers (survivin, Bcl-2 family, Livin, and $\mathrm{Bax}$ ), inactivating mutations in tumor suppressors (TP53 and RB1), cell cycle regulatory proteins (p16, p21, $\mathrm{p} 27$, cyclin $\mathrm{E} 1$, and $\mathrm{p} 27$ ), proliferation and angiogenesis biomarkers (Ki-67, VEGF, HIF-1 $\alpha$, and microvessel density), cell signaling pathway biomarkers (ErbB and FGFR family members), tumor cell invasion biomarkers (E-cadherin and $\mathrm{N}$-cadherin), and DDR gene mutations (APE1, ERCC1, ERCC2, XRCC1, XRCC3, hOGG1, $\mathrm{XPD}$, and XPG) have been suggested as prognosticators of outcome and predictors of response to therapy in MIBC $(34,36,43-46)$. A recent study implicated the activationinduced deaminase (AID)/apolipoprotein B mRNA editing catalytic polypeptide-like (APOBEC) family of cytidine deaminases, particularly APOBEC $3 \mathrm{~A}$ and APOBEC $3 \mathrm{~B}$, in cancer development and clonal evolution of cancer (47). Glaser et al. used BC datasets to examine the mutational signature of APOBEC and found that tumors enriched for APOBEC mutagenesis survived longer and were more likely to harbor mutations in DDR genes and chromatinmodifying genes; by contrast, APOBEC-low tumors were more likely to harbor mutations in FGFR3 and RAS 


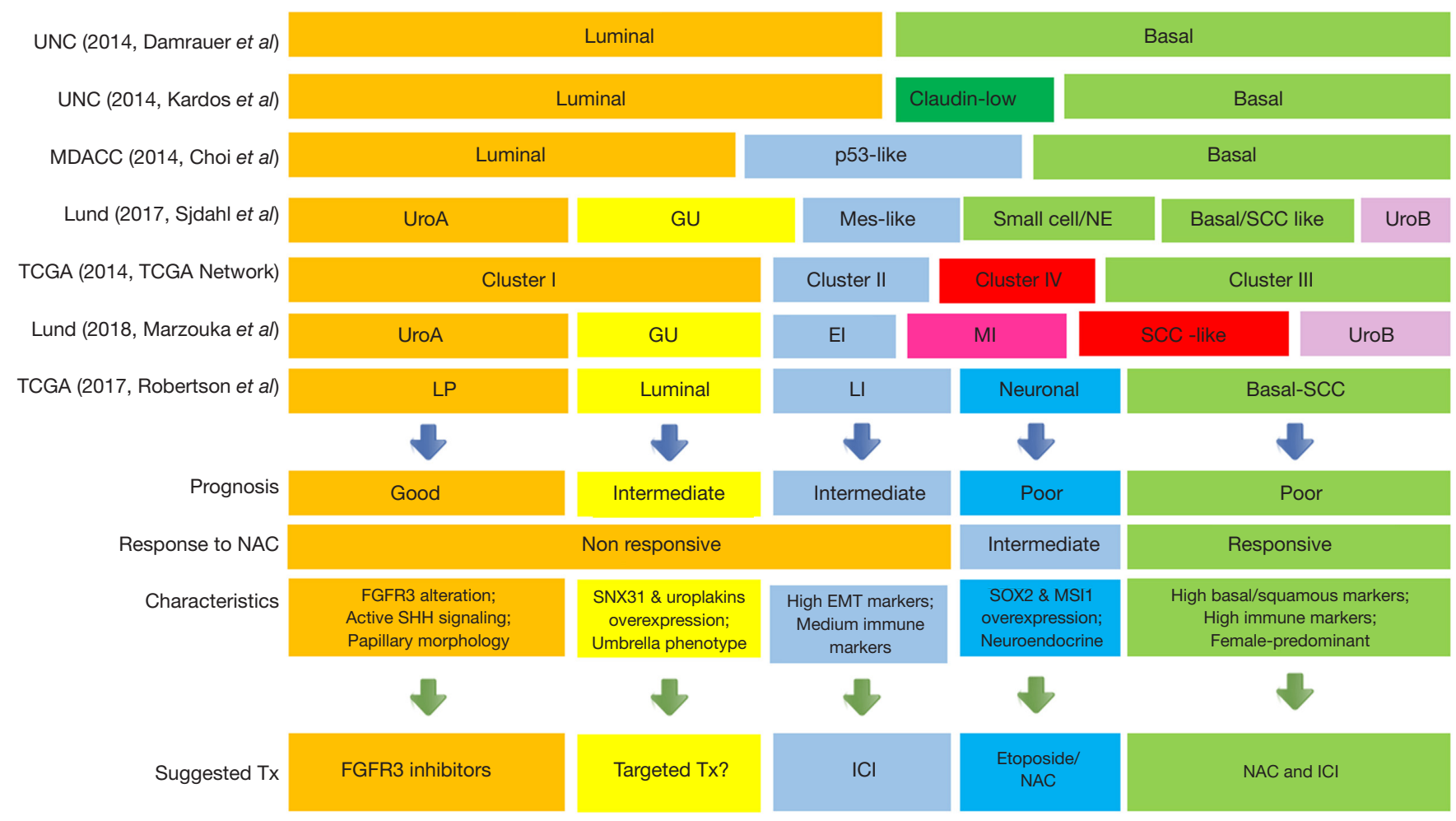

Figure 3 Molecular subtype classification based on gene expression profiling of MIBC. The schematic illustrates the taxonomic classifications; however, the interrelationships between each taxonomic classification are somewhat unclear. UNC, University of North Carolina; MDACC, MD Anderson Cancer Center; GU, genomically unstable; Mes, mesenchymal; NE, neuroendocrine; SCC, squamous cell carcinoma; EI, epithelial infiltrated; MI, mesenchymal infiltrated; LP, luminal-papillary; LI, luminal-infiltrated; ICI, immune checkpoint inhibitor; NAC, neoadjuvant chemotherapy.

family oncogenes (48). Expression of APOBEC3A and APOBEC3B correlates with mutation burden, regardless of the molecular subtype of the bladder tumor (48). The authors proposed a working model of mutagenesis and the immune response in $\mathrm{BC}$, in which accumulated mutations in DDR genes or genes responsible for chromatin regulation may expose single strand DNA to APOBEC3A and APOBEC $3 \mathrm{~B}$; this results in high levels of APOBECmediated mutagenesis and development of a hypermutation phenotype, with a subsequent neoantigen burden, immune response, and survival benefit (48).

However, it is unlikely that any single marker adequately characterizes the potential behavior of MIBC to allow reliable treatment conclusions (36). High-throughput microarray technology enables identification of progression markers and prediction of disease outcome in those with high grade T1 BC and MIBC (49-53). Gene signatures that predict chemo-responsiveness in adjuvant and neoadjuvant settings have been proposed (4,54). In addition, transcriptome profiling enables classification of BC into molecular subtypes; this mean that a patient's cancer can be stratified more precisely according to prognosis and therapeutic options (3).

\section{Molecular classification of MIBC}

Several groups describe different but overlapping, primarily RNA-based, molecular classifications of MIBC that resemble the taxonomy of breast cancer (Figure 3). In general, markers used to classify BCs into the two major groups reflect the expression signature of normal basal (CK5/6 and CK14) and intermediate/luminal urothelial cell layers (uroplakins, CK20, and GATA3) (55). In a chemotherapy-naive setting, tumors belonging to a basal MIBC subtype were more aggressive, with shorter survival times, than luminal cancers. Meanwhile, basal cancers 
were more sensitive to cisplatin-based chemotherapy, and patients with this form of disease appeared to gain more survival benefit from frontline chemotherapy than those with a luminal subtype (55). Several groups used whole transcriptome expression profiling to classify BC into distinct subtypes $(35,41,56-59)$. Overall, various molecular classification systems reached broad agreement about the characteristics of basal and luminal subtypes. A pioneering report by Lund group described five molecular subtypes of BC (Uro A, genomically unstable, infiltrated, squamous cell carcinoma-like, and Uro B) and then refined and extended this to six subtypes: urothelial-like, genomically unstable, epithelial infiltrated, SCC-like/Mes-like, SCC-like/UroB, and Sc/NE-like $(35,60)$. The UNC (University of North Carolina) group also presented a two-tiered system (basal and luminal subtypes), later extended to three classes upon identification of the claudin-low subtype $(61,62)$. MDACC (MD Anderson Cancer Center) presented a three-tiered system (basal, luminal, p53-like), further extended to include five classes: luminal, luminal-p53, basal, basal-p53 classes, and "double negative" $(55,58)$. An earlier TCGA study produced a four-class system with groups referred to as I-IV (I and II: luminal; III and IV: basal); this was based on data from an interim cohort of 131 TCGA tumors, the subtypes of which were then refined and extended to five classes using a full TCGA cohort of 412 (57). This study generated basal (basal-squamous) and luminal subtypes (luminalinfiltrated, luminal, and luminal-papillary), which are similar to their original subtypes, and identified a new subtype (neuronal) that expressed neuroendocrine tumor markers (63). A previously published classification system segregated MIBC into 2-7 molecular subtypes using different subtype names (3). Thus, different studies have generated many different subtypes; it is this diversity of molecular subtyping that has hindered its transfer into clinical practice (3). Recently, a consensus classification unifying these different subtypes was proposed to facilitate clinical application (3). The Bladder Cancer Molecular Taxonomy Group examined 1750 MIBC transcriptomes and performed a network-based analysis of six independent MIBC classification systems; as a result, they identified a consensus set comprising six molecular classes: luminal-papillary (24\%), luminal nonspecified (8\%), luminal unstable (15\%), stroma-rich (15\%), basal/squamous (35\%), and neuroendocrine-like (3\%) (3). Ongoing studies are examining the relevance of these molecular subtypes to $\mathrm{BC}$ histopathological variants and to upper urinary tract urothelial cancer (64-66).
It is important to note that different molecular subtypes of $\mathrm{BC}$ are not equally sensitive to chemotherapy and immune checkpoint blockade; indeed, these subtypes respond differently to radiation, with or without hypoxia modulation (Figure 3) $(58,63,67,68)$. Choi et al. report that patients with the p53-like subtype show a particularly low response rate to NAC; the p53-like subtype is characterized by fibroblast infiltration and a senescent signature (58). Robertson and colleagues provide insight into mutational profiles that have prognostic value and establish a framework for associating distinct tumor subtypes with certain clinical options (63). The luminal-papillary subtype has a low risk for progression, with preliminary data suggesting that it is unlikely to respond to cisplatin-based NAC (69). The luminal-infiltrated subtype in patients with metastatic or unresectable $\mathrm{BC}$ responds to immune checkpoint therapy with atezolizumab and may be resistant to cisplatin-based chemotherapy (63). Luminal-papillary tumors show high FGFR3 expression and harbor genomic FGFR3-activating mutations in addition to being resistant to immune cell infiltration (69). This implies that FGFR3-targeted therapy (e.g., erdafitinib) could be advantageous in this subtype, and that checkpoint blockade by itself would likely have little benefit (63). The basal-squamous subtype is characterized by squamous differentiation and high expression of CD274 (PD-L1) and CTLA4 (63). Thus, cisplatin-based NAC and immune checkpoint therapies are appropriate therapeutic options. Another gene expression analysis study identified a neuroendocrine subtype with a particularly poor prognosis, even in the absence of typical histologic neuroendocrine features (70). It is unclear whether these tumors should be regarded as neuroendocrine tumors and treated with cisplatin/etoposide (69). Interestingly, a recent analysis demonstrated that neuronal tumors in the TCGA were associated with high response rates when patients were treated with atezolizumab (anti-PDL1) in the ImVigor 210 trial (71).

\section{Intra-tumor heterogeneity in MIBC}

Inter-tumor heterogeneity explains differences in responses to standard chemotherapy and immune checkpoint inhibitors. Intra-tumor heterogeneity is based on the presence of different histological types within the same tumor type from a single patient; an example is the mixed histology observed in urothelial and histologic variants of BC (72). Intra-tumor heterogeneity may also 


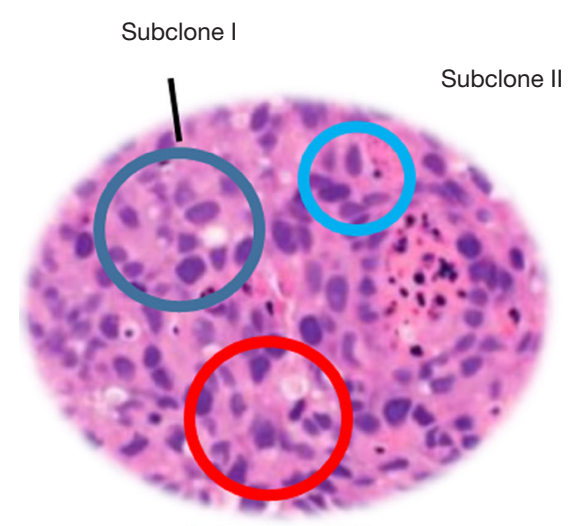

Subclone III

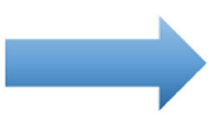

1st line therapy

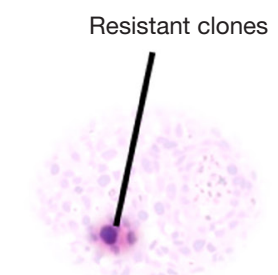

Tumor shrinkage, but "subclinical" recurrence
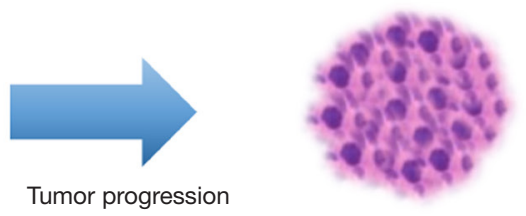

"Clinical" recurrence

Figure 4 The clinical implications of tumor heterogeneity. First-line treatment can kill dominant clones; however, resistant clones persist and drive tumor progression. Resistant clones may metastasize after initial treatment. A new diagnosis is needed to identify an appropriate second-line treatment.

explain why some patients show an initial response to therapy, followed by recurrence and progression of a resistant clone (Figure 4) (41).

Spatial intra-tumor heterogeneity is characterized by frequent co-occurrence of conventional UCC alongside histologic variants (e.g., squamous, glandular, micropapillary, nested, plasmacytoid, and sarcomatoid of BC (73). Recent data suggest that molecular subtype within multiregional tumor samples may be heterogeneous $(65,74)$. For example, Warrick et al. found that different areas within the same tumor often show different molecular patterns (59). They used the Lund university classification system to categorize 83 histologically variant BC tumors into different molecular subtypes. In their cohort, they showed that 39\% of tumors with multiple histologies demonstrated molecular heterogeneity. This was predominant in basal-squamous subtypes, which co-occurred with either urothelial-like or genomically unstable carcinomas (59). However, they did not observe co-occurrence of genomically unstable and urothelial-like subtypes in the same patient. This information helps us to understand how BC develops variable histology. The findings suggest the concept of intratumoral molecular heterogeneity in BC patients, and raise concerns about sampling errors in laboratory tests that guide therapy choices based on molecular subtyping results.

It is also important to understand temporal intra-tumor heterogeneity signatures after treatment; this is because platinum-based chemotherapy and immunotherapy are very likely to alter the biological and clinical behavior of a tumor, which will affect molecular subtyping results $(75,76)$. Molecular alterations induced by chemotherapy are poorly characterized; however, a study shows that significant alterations occur at both the genomic and transcriptomic levels (77). Faltas et al. identified a marked shift in the mutational landscape of UCC after chemotherapy, which may be driven by chemotherapyinduced selection pressure (78). Early studies of molecular subtypes suggest that NAC induces subtype switching, indicating that NAC either induces phenotypic changes at the transcriptome level or selects one subtype over another in a mixed, heterogeneous tumor $(58,77)$. Liu et al. used whole exome sequencing to analyze matched pre- and post-NAC samples from 30 patients with MIBC (79). They noted that a significant proportion of sub-clonal mutations are unique to the matched preor post-treatment tumor, suggesting chemotherapyinduced and/or spatial heterogeneity (79). The authors demonstrated that tumor heterogeneity in post-NAC tumors is associated with poor overall survival; in addition, they observed other alterations in genes responsible for regulating the cell cycle and immune checkpoints in post-treatment tumors. Recently, Seiler et al. expanded our knowledge about the lack of response of MIBC to cisplatin-based NAC; they suggested different molecular subtypes to explain the biology of these tumors (77). They matched the gene expression profiles of 116 tumor samples from patients with residual disease at two time points: pre- (TUR specimens) and post-NAC (RC 
specimens). They identified four consensus clusters in the post-NAC cohort, with residual disease characterized as a CC1-basal, CC2-luminal, CC3-immune subtype, or CC4scar like subtype. Pre- and post-NAC patients with basal or luminal subtypes had a better prognosis than those with claudin-low or luminal-infiltrated tumors, whereas postNAC samples with MDA p53-like and Lund-infiltrated subtypes had a better prognosis. Post-NAC molecular subtypes differed from pre-NAC subtypes, with post-NAC samples comprising fewer luminal subtypes but more basal, p53, and infiltrated subtypes. Thirty-four percent of cases showed a marked change in molecular subtype between pre- and post-NAC, with the CC3-immune subtype losing luminal and basal markers and showing greater immune activity. Of the tumors that lacked immune infiltration preNAC, 32\% showed immune infiltration post-NAC (77). A study by Seiler et al. compared pre- and post-NAC MIBC samples; the results are an important step toward identifying molecular signatures of cisplatin resistance; as such, it may form the basis for future clinical studies. Identifying the molecular subtype of post-NAC tumors will help direct the use of adjuvant or salvage therapy in MIBC patients and may play a crucial role in improving overall outcomes (77).

\section{Emerging molecular biomarkers for personalized cancer therapy for MIBC}

During cancer progression and treatment, multiple subclonal populations of tumor cells compete with one another. Selective pressure drives the emergence of predominant sub-clones; these sub-clones replicate and spread more efficiently and are resistant to treatment (Figure 4) $(80,81)$. Therefore, longitudinal tracking of genetic and epigenetic alterations is required to assess the molecular heterogeneity of primary and secondary tumors, and to identify genetic determinants for targeted therapy (82). Currently, the molecular characteristics of solid tumors are identified using tissue obtained at surgery or by biopsy. However, tissue-based tumor profiles are subject to sampling bias and capture a snapshot of tumor heterogeneity; in addition, repeated sampling is not possible $(80,83)$. Liquid biopsy encompasses myriad assays that measure tumor-derived cells or their products in blood and other body fluids that, in general, may be obtained serially and easily with minimal risk to the patient (82). Liquid biopsies are very promising because they can record and monitor disease status in real time; they can also predict prognosis, recurrence, therapy response, and resistance, all without the need for invasive interventions (33).

During the initial stages of metastatic progression, cancer cells detached from the primary tumor invade the lymphatics and systemic circulation; these cells are called circulating tumor cells (CTCs) $(84,85)$. RT-PCR-based protocols that utilize PCR primers that target tumor-specific genes (e.g., cytokeratin-20, uroplakin II, and epidermal growth factor receptor) are quite non-specific $(85,86)$. This has led to the development of the CellSearch assay, which is a semiautomated, standardized enrichment and detection system based on magnetically labeled anti-EpCAM antibodies; these antibodies bind to CTCs that are then visualized and counted under a digital fluorescence microscope. This assay system has been adapted to detect circulating urothelial CSCs in the peripheral blood of patients with metastatic and non-metastatic urothelial cancers (87). The IsoFlux system also uses immunomagnetic capture of CTCs based on EpCAM expression; cells are captured in a microfluidic device to increase specificity for CTCs and minimize white blood cell carry over (88). Alva et al. compared the sensitivity of CTC detection using the CellSearch assay with that using the IsoFlux method (88). They found that the IsoFlux method was more sensitive than CellSearch and enables molecular profiling of recovered cells via nextgeneration sequencing (NGS) (88). Detection of CTCs may flag an early step of the metastatic process, which may be a precursor to establishment of clinically undetectable micrometastatic foci that will grow into clinically apparent metastases $(85,89)$. However, clinical reports evaluating molecular detection of CTCs yield contradictory and inconclusive results. Some studies show that detection of CTCs may be associated with more advanced disease, whereas others fail to detect any association at all $(85,86$, 89-94). Msaouel et al. performed a meta-analysis to examine the diagnostic and prognostic value of CTC detection in BC and/or UCC; the analysis included 21 studies that investigated the presence of CTCs in peripheral blood (85). The findings revealed that the overall sensitivity of CTC detection assays was $35.1 \%$ (95\% CI, 32.4-38\%) and that the specificity and positive- and negative-likelihood ratios were $89.4 \%$ (95\% CI, 87.2-91.3\%), 3.77 (95\% CI, $1.95-7.30$ ), and 0.72 (95\% CI, 0.64-0.81), respectively (85). They also suggested that CTC-positive patients were significantly more likely to have advanced (stage III-IV) disease than CTC-negative patients (OR, 5.05; 95\% CI, 2.49-10.26) (85). In 2017, Zhang et al. published a metaanalysis of 30 published studies examining the impact of CTCs in UCC; they found a significant association between 
CTC-positivity and tumor stage ( $\leq$ II vs. III, IV) (OR 4.60, 95\% CI, 2.34-9.03), histological grade (I, II vs. III) (OR 2.91, 95\% CI, 1.92-4.40), metastasis (OR 5.12, 95\% CI, 3.47-7.55), and regional lymph node metastasis (OR 2.47, 95\% CI, 1.75-3.49). CTC-positivity was also associated significantly with poor overall survival (OS) (HR 3.98, 95\% CI, 2.20-7.21), progression/disease-free survival (PFS/ DFS) (HR 2.22, 95\% CI, 1.80-2.73), and cancer-specific survival (CSS) (HR 5.18, 95\% CI, 2.21-12.13) (95). They also revealed that the overall sensitivity and specificity of CTC detection assays were 0.35 (95\% CI, 0.28-0.43) and 0.97 (95\% CI, 0.92-0.99), respectively (95). CTC detection assays fail to identify almost two thirds of patients; thus they have limited diagnostic sensitivity for BC. It is unclear whether CTCs are truly rare in BC, or whether many are missed by EpCAM-based technology (96). One possible reason for such a failure is that metastatic cells lose EpCAM expression as they undergo EMT; therefore, EpCAMbased approaches fail to detect subpopulations of CTCs with intermediate or pure mesenchymal features. Recently, alternative methods that allow EpCAM-independent CTC detection, such as the AdnaTest platform and ScreenCell Cyto devices, have been explored with respect to BC. Although a variety of methods have been used to improve sensitivity for detecting CTCs, further technological advances will be necessary before detecting CTCs becomes clinically relevant in patients with BC. Due to the limited diagnostic sensitivity and high overall specificity of CTC detection, the technique may not be sufficiently accurate for initial screening tests; however, detecting CTCs at different time points may allow real time surveillance of dynamic changes in disease status and, crucially, increase our understanding of the metastatic cascade, thereby facilitating design of novel targeted therapy approaches (33).

Apoptotic or necrotic cancer cells release circulating cell-free DNA (cfDNA) fragments [known as circulating tumor DNA (ctDNA)], mRNAs, long non-coding RNAs, and microRNAs, as well as proteins, peptides, metabolites, and extracellular vesicles (exosomes, microvesicles, and oncosomes) (84). Many cancer-related molecular entities, including single nucleotide variations, copy number aberrations, aberrations in methylation, and gene expression alterations, have been detected in cell-free nucleic acids (97). An in-depth review of each of these markers is not possible here. Therefore, we will focus on ctDNA in blood and urine. Circulating ctDNA comprises cfDNA shed by tumor cells into the circulatory system. Thus, ctDNA contains tumor-specific genomic variants that can be utilized as unique genetic signatures or biomarkers (98). Analysis of ctDNA has several potential advantages over solid tumor biopsy samples, making it useful for tumor molecular profiling (98). Tumors are temporally and spatially heterogeneous; therefore, a tissue biopsy may only provide a "snapshot" of one region within one tumor at one point in time (98). It is likely that ctDNA likely derives from all tumor sites; therefore, ctDNA samples are likely to provide a more representative snapshot of an individual cancer than a biopsy sample. This is because tumor cell clones derived from the primary, micro-, and macro-metastatic deposits are present in a single sample, allowing more accurate monitoring of a patient's disease burden and progression in real time by detecting DNA that characterizes intratumor and inter-tumor heterogeneities $(98,99)$. The major challenge for ctDNA diagnostics is to identify and track rare mutated DNA fragments among thousands of wildtype DNA copies (98). Initially, allele-specific primers, commonly used for conventional PCR and pyrosequencing, were used to detect and measure the percentage of specific mutations in cfDNA present in liquid biopsy samples; however, restriction to specific mutations coupled with low sensitivity has limited these techniques (33). The situation was improved by using quantitative PCR and deep sequencing technologies such as NGS or digital droplet polymerase PCR (ddPCR) (33). Nagy et al. used a 70-gene NGS panel to perform cfDNA analysis of patients with advanced BC and detected clinically significant levels of ctDNA in $86 \%$ of patients; within these fragments, they detected somatic mutations in TP53, kinase genes, epigenetic modifiers, and genes that regulate the cell cycle (100). A recent study by Grivas et al. evaluated 124 patients with advanced UCC who underwent cfDNA analysis using a 73-gene sequencing panel. They detected at least one genomic alteration in 112 patients $(90.3 \%)$, with a median four alterations per sample. The authors suggested that alterations in BRCA1 and RAF1 were associated with worse OS and PFS (101). A recent breakthrough technology study by Gootenberg et al. combined the Cas13a enzyme with isothermal amplification to establish a clustered regularly interspaced short palindromic repeats (CRISPR)-based diagnostic system named SHERLOCK (specific highsensitivity enzymatic reporter unlocking). This assay enables rapid detection of DNA or RNA with attomolar sensitivity and single-base mismatch specificity (102). Urinary ctDNA is derived from tumor cells that are shed directly into the urinary tract, making it highly representative of the tumor 
genome from which the ctDNA arises; in addition, it is present at higher concentrations than in serum (98). Limited data are available on the clinical utility of urine ctDNA in MIBC because most urine ctDNA studies have focused on initial diagnostic and prognostic makers of NMIBC. Kim and colleagues used RT-PCR to examine the diagnostic utility of urinary topoisomerase-II alpha (TopoIIA) cfDNA in $83 \mathrm{BC}$ samples, and found a significant difference in urinary TopoIIA ctDNA levels between NMIBC and MIBC (103).

ScRNA-seq analysis is an efficient method of identifying heterogeneous gene expression and the characteristics of each subpopulation within a tumor at a single cell level. Most transcriptome studies to date are carried out on a 'population level', usually averaging the transcriptomes of millions of cells (104). This bulk-cell DNA sequencing approach is limited with respect to identifying transcriptomic inter-tumor heterogeneity because it only infers the presence of clones, rather than detecting individual cells directly (105). Single cell transcriptomics provides an opportunity to better understand transcriptional stochasticity and cellular heterogeneity, both of which are important for maintaining cell function, disease progression, and treatment responses; these details are often masked in bulk-cell studies (105,106-108). Zhang and colleagues performed scRNA-seq on squamous cell carcinoma of the bladder and characterized heterogeneous gene expression profiles; they then compared their transcriptional, functional, and genetic diversity (106). Recently, Tanaka et al. used the scRNA-seq system to screen urinary BCs and revealed a dynamic shift in ITH before and after development of platinum-resistance (109). They identified a novel gene, COX7B, which was associated with platinum-resistance, and a surrogate marker, CD63, which can identify low COX7B-expressing sub-clones (109).

\section{Conclusions}

New genomic techniques provide insight into the marked genetic complexity and the vast inter- and intra-tumor heterogeneity of MIBC. Over the last decade, RNAbased molecular subtyping has identified distinct or partially overlapping molecular classifications of MIBC. Recent studies show that molecular stratification of MIBC is of clinical importance, suggesting that responses to chemotherapy and immunotherapy may be increased for specific MIBC subtypes. Tumor heterogeneity is not a fixed state; rather, it should be considered as a dynamic ecosystem that evolves as the tumor progresses and is modulated strongly by therapeutic pressure. Microenvironmentdriven tumor heterogeneity and plasticity also play a key role in tumor progression and resistance to treatment. Real time approaches to molecular monitoring of evolving genetic clones responsible for tumor progression and drug resistance remain a challenging task. In this context, liquid biopsy has emerged as a highly promising tool for cancer screening, surveillance, disease monitoring, and identification of new therapeutic targets. Recent developments in tumor-specific, highly sensitive assays such as NGS, ddPCR, or CRISPR enzyme-based diagnostic platforms for molecular profiling of CTCs at single cell resolution, and analysis and detection of cell-free nucleic acids (ctDNA, miRNAs) and extracellular vesicles in serum and urine, suggest that liquid biopsy may have clinical applications for MIBC. Despite the tremendous potential of liquid biopsy, real-world application in MIBC is hindered by poor specificity and sensitivity, lack of standardization, and poor reproducibility. Clearly, further investigations and large-scale validation studies are needed before liquid biopsy can be applied successfully in routine clinical practice.

\section{Acknowledgments}

Funding: This research was supported by the International Science and Business Belt Program through the Ministry of Science, ICT, and Future Planning (2017K000490), and the Basic Science Research Program through the National Research Foundation of Korea (NRF), funded by the Ministry of Education (2017R1D1A1B03033629).

\section{Footnote}

Provenance and Peer Review: This article was commissioned by the Guest Editors (Ja Hyeon Ku, Ho Kyung Seo, Seok Ho Kang) for the series "Muscle-Invasive Bladder Cancer" published in Translational Andrology and Urology. The article has undergone external peer review.

Conflicts of Interest: All authors have completed the ICMJE uniform disclosure form (available at http://dx.doi. org/10.21037/tau.2020.03.13). The series "Muscle-Invasive Bladder Cancer" was commissioned by the editorial office without any funding or sponsorship. The authors have no other conflicts of interest to declare.

Ethical Statement: The authors are accountable for all 
aspects of the work in ensuring that questions related to the accuracy or integrity of any part of the work are appropriately investigated and resolved.

Open Access Statement: This is an Open Access article distributed in accordance with the Creative Commons Attribution-NonCommercial-NoDerivs 4.0 International License (CC BY-NC-ND 4.0), which permits the noncommercial replication and distribution of the article with the strict proviso that no changes or edits are made and the original work is properly cited (including links to both the formal publication through the relevant DOI and the license). See: https://creativecommons.org/licenses/by-nc-nd/4.0/.

\section{References}

1. 1. Chen C, Qi XJ, Cao YW, et al. Bladder Tumor Heterogeneity: The Impact on Clinical Treatment. Urol Int 2015;95:1-8.

2. Kang HW, Kim WJ, Yun SJ. The role of the tumor microenvironment in bladder cancer development and progression. Transl Cancer Res 2017:S744-S58.

3. Kamoun A, de Reyniès A, Allory Y, et al. A Consensus Molecular Classification of Muscle-invasive Bladder Cancer. Eur Urol 2020;77:420-33.

4. Kim WT, Kim J, Yan C, et al. S100A9 and EGFR gene signatures predict disease progression in muscle invasive bladder cancer patients after chemotherapy. Ann Oncol 2014;25:974-9.

5. Robins D, Matulay J, Lipsky M, et al. Outcomes Following Clinical Complete Response to Neoadjuvant Chemotherapy for Muscle-invasive Urothelial Carcinoma of the Bladder in Patients Refusing Radical Cystectomy. Urology 2018;111:116-21.

6. Wezel F, Vallo S, Roghmann F. Do we have biomarkers to predict response to neoadjuvant and adjuvant chemotherapy and immunotherapy in bladder cancer? Transl Androl Urol 2017;6:1067-80.

7. Marcq G, Jarry E, Ouzaid I, et al. Contemporary best practice in the use of neoadjuvant chemotherapy in muscle-invasive bladder cancer. Ther Adv Urol 2019;11:1756287218823678.

8. Mohammed AA, El-Tanni H, El-Khatib HM, et al. Urinary Bladder Cancer: Biomarkers and Target Therapy, New Era for More Attention. Oncol Rev 2016;10:320.

9. Mendiratta P, Grivas P. Emerging biomarkers and targeted therapies in urothelial carcinoma. Ann Transl Med
2018;6:250.

10. Bedard PL, Hansen AR, Ratain MJ, et al. Tumour heterogeneity in the clinic. Nature 2013;501:355-64.

11. Dirkse A, Golebiewska A. Stem cell-associated heterogeneity in Glioblastoma results from intrinsic tumor plasticity shaped by the microenvironment. Nat Commun 2019;10:1787.

12. Fisher R, Pusztai L, Swanton C. Cancer heterogeneity: implications for targeted therapeutics. Br J Cancer 2013;108:479-85.

13. Zhang J, Spath SS, Marjani SL, et al. Characterization of cancer genomic heterogeneity by next-generation sequencing advances precision medicine in cancer treatment. Precis Clin Med 2018;1:29-48.

14. Herceg Z, Hainaut P. Genetic and epigenetic alterations as biomarkers for cancer detection, diagnosis and prognosis. Mol Oncol 2007;1:26-41.

15. Rich JN. Cancer stem cells: understanding tumor hierarchy and heterogeneity. Medicine (Baltimore) 2016;95:S2-7.

16. Prasetyanti PR, Medema JP. Intra-tumor heterogeneity from a cancer stem cell perspective. Mol Cancer 2017;16:41.

17. Nowell PC. The clonal evolution of tumor cell populations. Science 1976;194:23-8.

18. Asatryan AD, Komarova NL. Evolution of genetic instability in heterogeneous tumors. J Theor Biol 2016;396:1-12.

19. Diaz-Cano SJ. Tumor heterogeneity: mechanisms and bases for a reliable application of molecular marker design. Int J Mol Sci 2012;13:1951-2011.

20. Gerdes MJ, Sood A, Sevinsky C, et al. Emerging understanding of multiscale tumor heterogeneity. Front Oncol 2014;4:366.

21. Ayob AZ, Ramasamy TS. Cancer stem cells as key drivers of tumour progression. J Biomed Sci 2018;25:20.

22. Vinogradov S, Wei X. Cancer stem cells and drug resistance: the potential of nanomedicine. Nanomedicine (Lond) 2012;7:597-615.

23. Plaks V, Kong N, Werb Z. The cancer stem cell niche: how essential is the niche in regulating stemness of tumor cells? Cell Stem Cell 2015;16:225-38.

24. Ho PL, Kurtova A, Chan KS. Normal and neoplastic urothelial stem cells: getting to the root of the problem. Nat Rev Urol 2012;9:583-94.

25. Lee G, Hall RR 3rd, Ahmed AU. Cancer Stem Cells: Cellular Plasticity, Niche, and its Clinical Relevance. J 
Stem Cell Res Ther 2016;6.

26. Poli V, Fagnocchi L, Zippo A. Tumorigenic Cell Reprogramming and Cancer Plasticity: Interplay between Signaling, Microenvironment, and Epigenetics. Stem Cells Int 2018;2018:4598195.

27. Raja R, Pandey A, Kumar P. Epithelial to mesenchymal plasticity: role in cancer progression. Front Biosci (Landmark Ed) 2020;25:838-73.

28. Wahl GM, Spike BT. Cell state plasticity, stem cells, EMT, and the generation of intra-tumoral heterogeneity. NPJ Breast Cancer 2017;3:14.

29. Chew V, Toh HC, Abastado JP. Immune microenvironment in tumor progression: characteristics and challenges for therapy. J Oncol 2012;2012:608406.

30. Wang M, Zhao J, Zhang L, et al. Role of tumor microenvironment in tumorigenesis. J Cancer 2017;8:761-73.

31. Prieto-Vila M, Takahashi RU, Usuba W, et al. Drug Resistance Driven by Cancer Stem Cells and Their Niche. Int J Mol Sci 2017. doi: 10.3390/ijms18122574.

32. Emens LA, Silverstein SC, Khleif S, et al. Toward integrative cancer immunotherapy: targeting the tumor microenvironment. J Transl Med 2012;10:70.

33. Lodewijk I, Duenas M. Liquid Biopsy Biomarkers in Bladder Cancer: A Current Need for Patient Diagnosis and Monitoring. Int J Mol Sci 2018. doi: 10.3390/ ijms19092514.

34. Liebert M, Seigne J. Characteristics of invasive bladder cancers: histological and molecular markers. Semin Urol Oncol 1996;14:62-72.

35. Sjodahl G, Lauss M, Lovgren K, et al. A molecular taxonomy for urothelial carcinoma. Clin Cancer Res 2012;18:3377-86.

36. Soria F, Krabbe LM, Todenhofer T, et al. Molecular markers in bladder cancer. World J Urol 2019;37:31-40.

37. Cheng L, Zhang S, MacLennan GT, et al. Bladder cancer: translating molecular genetic insights into clinical practice. Hum Pathol 2011;42:455-81.

38. Ye F, Wang L, Castillo-Martin M, et al. Biomarkers for bladder cancer management: present and future. Am J Clin Exp Urol 2014;2:1-14.

39. Baffa R, Letko J, McClung C, et al. Molecular genetics of bladder cancer: targets for diagnosis and therapy. J Exp Clin Cancer Res 2006;25:145-60.

40. Audenet F, Attalla K, Sfakianos JP. The evolution of bladder cancer genomics: What have we learned and how can we use it? Urol Oncol 2018;36:313-20.
41. da Costa JB, Gibb EA, Nykopp TK, et al. Molecular tumor heterogeneity in muscle invasive bladder cancer: Biomarkers, subtypes, and implications for therapy. Urol Oncol 2018. [Epub ahead of print].

42. Stadler WM, Lerner SP, Groshen S, et al. Phase III study of molecularly targeted adjuvant therapy in locally advanced urothelial cancer of the bladder based on p53 status. J Clin Oncol 2011;29:3443-9.

43. Groenendijk FH, de Jong J, Fransen van de Putte EE, et al. ERBB2 Mutations Characterize a Subgroup of Muscleinvasive Bladder Cancers with Excellent Response to Neoadjuvant Chemotherapy. Eur Urol 2016;69:384-8.

44. Van Allen EM, Mouw KW, Kim P, et al. Somatic ERCC2 mutations correlate with cisplatin sensitivity in muscle-invasive urothelial carcinoma. Cancer Discov 2014;4:1140-53.

45. Takata R, Katagiri T, Kanehira M, et al. Predicting response to methotrexate, vinblastine, doxorubicin, and cisplatin neoadjuvant chemotherapy for bladder cancers through genome-wide gene expression profiling. Clin Cancer Res 2005;11:2625-36.

46. Cheng T, Roth B, Choi W, et al. Fibroblast growth factor receptors-1 and -3 play distinct roles in the regulation of bladder cancer growth and metastasis: implications for therapeutic targeting. PLoS One 2013;8:e57284.

47. Rebhandl S, Huemer M, Greil R, et al. AID/APOBEC deaminases and cancer. Oncoscience 2015;2:320-33.

48. Glaser AP, Fantini D, Wang Y, et al. APOBEC-mediated mutagenesis in urothelial carcinoma is associated with improved survival, mutations in DNA damage response genes, and immune response. Oncotarget 2018;9:4537-48.

49. Kim WJ, Kim EJ, Kim SK, et al. Predictive value of progression-related gene classifier in primary non-muscle invasive bladder cancer. Mol Cancer 2010;9:3.

50. Kang HW, Seo SP, Byun YJ, et al. Molecular Progression Risk Score for Prediction of Muscle Invasion in Primary T1 High-Grade Bladder Cancer. Clin Genitourin Cancer 2018;16:274-80.

51. Smith SC, Baras AS, Dancik G, et al. A 20-gene model for molecular nodal staging of bladder cancer: development and prospective assessment. Lancet Oncol 2011;12:137-43.

52. Seiler R, Lam LL, Erho N, et al. Prediction of Lymph Node Metastasis in Patients with Bladder Cancer Using Whole Transcriptome Gene Expression Signatures. J Urol 2016;196:1036-41.

53. Blaveri E, Simko JP, Korkola JE, et al. Bladder cancer outcome and subtype classification by gene expression. 
Clin Cancer Res 2005;11:4044-55.

54. Plimack ER, Dunbrack RL, Brennan TA, et al. Defects in DNA Repair Genes Predict Response to Neoadjuvant Cisplatin-based Chemotherapy in Muscle-invasive Bladder Cancer. Eur Urol 2015;68:959-67.

55. Dadhania V, Zhang M, Zhang L, et al. Meta-Analysis of the Luminal and Basal Subtypes of Bladder Cancer and the Identification of Signature Immunohistochemical Markers for Clinical Use. EBioMedicine 2016;12:105-17.

56. Marzouka NA, Eriksson P, Rovira C, et al. A validation and extended description of the Lund taxonomy for urothelial carcinoma using the TCGA cohort. Sci Rep 2018;8:3737.

57. Comprehensive molecular characterization of urothelial bladder carcinoma. Nature 2014;507:315-22.

58. Choi W, Porten S, Kim S, et al. Identification of distinct basal and luminal subtypes of muscle-invasive bladder cancer with different sensitivities to frontline chemotherapy. Cancer Cell 2014;25:152-65.

59. Warrick JI, Sjodahl G, Kaag M, et al. Intratumoral Heterogeneity of Bladder Cancer by Molecular Subtypes and Histologic Variants. Eur Urol 2019;75:18-22.

60. Aine M, Eriksson P, Liedberg F, et al. Biological determinants of bladder cancer gene expression subtypes. Sci Rep 2015;5:10957.

61. Damrauer JS, Hoadley KA, Chism DD, et al. Intrinsic subtypes of high-grade bladder cancer reflect the hallmarks of breast cancer biology. Proc Natl Acad Sci U S A 2014;111:3110-5.

62. Kardos J, Chai S, Mose LE, et al. Claudin-low bladder tumors are immune infiltrated and actively immune suppressed. JCI Insight 2016;1:e85902.

63. Robertson AG, Kim J, Al-Ahmadie H, et al. Comprehensive Molecular Characterization of MuscleInvasive Bladder Cancer. Cell 2018;174:1033.

64. Weyerer V, Weisser R, Moskalev EA. Distinct genetic alterations and luminal molecular subtype in nested variant of urothelial carcinoma. Histopathology 2019;75:865-75.

65. Al-Ahmadie H, Iyer G. Updates on the Genetics and Molecular Subtypes of Urothelial Carcinoma and Select Variants. Surg Pathol Clin 2018;11:713-23.

66. Moss TJ, Qi Y, Xi L, et al. Comprehensive Genomic Characterization of Upper Tract Urothelial Carcinoma. Eur Urol 2017;72:641-9.

67. McConkey DJ, Choi W, Shen Y, et al. A Prognostic Gene Expression Signature in the Molecular Classification of Chemotherapy-naive Urothelial Cancer is Predictive of Clinical Outcomes from Neoadjuvant Chemotherapy: A
Phase 2 Trial of Dose-dense Methotrexate, Vinblastine, Doxorubicin, and Cisplatin with Bevacizumab in Urothelial Cancer. Eur Urol 2016;69:855-62.

68. Seiler R, Ashab HAD, Erho N, et al. Impact of Molecular Subtypes in Muscle-invasive Bladder Cancer on Predicting Response and Survival after Neoadjuvant Chemotherapy. Eur Urol 2017;72:544-54.

69. Joshi M, Warrick JI, Yin M, et al. Need for a personalized approach for muscle invasive bladder cancer: role of tumor biology in response to neoadjuvant chemotherapy. Transl Androl Urol 2019;8:S99-103.

70. Sjodahl G, Eriksson P, Liedberg F, et al. Molecular classification of urothelial carcinoma: global mRNA classification versus tumour-cell phenotype classification. J Pathol 2017;242:113-25.

71. Kim J, Kwiatkowski D, McConkey DJ, et al. The Cancer Genome Atlas Expression Subtypes Stratify Response to Checkpoint Inhibition in Advanced Urothelial Cancer and Identify a Subset of Patients with High Survival Probability. Eur Urol 2019;75:961-4.

72. Chalasani V, Chin JL, Izawa JI. Histologic variants of urothelial bladder cancer and nonurothelial histology in bladder cancer. Can Urol Assoc J 2009;3:S193-8.

73. Porten SP, Willis D, Kamat AM. Variant histology: role in management and prognosis of nonmuscle invasive bladder cancer. Curr Opin Urol 2014;24:517-23.

74. Thomsen MBH, Nordentoft I, Lamy P, et al. Comprehensive multiregional analysis of molecular heterogeneity in bladder cancer. Sci Rep 2017;7:11702.

75. Dagogo-Jack I, Shaw AT. Tumour heterogeneity and resistance to cancer therapies. Nat Rev Clin Oncol 2018;15:81-94.

76. Rybinski B, Yun K. Addressing intra-tumoral heterogeneity and therapy resistance. Oncotarget 2016;7:72322-42.

77. Seiler R, Gibb EA, Wang NQ, et al. Divergent Biological Response to Neoadjuvant Chemotherapy in Muscleinvasive Bladder Cancer. Clin Cancer Res 2019;25:508293.

78. Faltas BM, Prandi D, Tagawa ST, et al. Clonal evolution of chemotherapy-resistant urothelial carcinoma. Nat Genet 2016;48:1490-9.

79. Liu D, Abbosh P, Keliher D, et al. Mutational patterns in chemotherapy resistant muscle-invasive bladder cancer. Nat Commun 2017;8:2193.

80. Siravegna G, Marsoni S, Siena S, et al. Integrating liquid biopsies into the management of cancer. Nat Rev Clin Oncol 2017;14:531-48. 
81. Di Meo A, Bartlett J, Cheng Y, et al. Liquid biopsy: a step forward towards precision medicine in urologic malignancies. Mol Cancer 2017;16:80.

82. Ramalingam N, Jeffrey SS. Future of Liquid Biopsies With Growing Technological and Bioinformatics Studies: Opportunities and Challenges in Discovering Tumor Heterogeneity With Single-Cell Level Analysis. Cancer J 2018;24:104-8.

83. Jamal-Hanjani M, Quezada SA, Larkin J, et al. Translational implications of tumor heterogeneity. Clin Cancer Res 2015;21:1258-66.

84. Palmirotta R, Lovero D, Cafforio P, et al. Liquid biopsy of cancer: a multimodal diagnostic tool in clinical oncology. Ther Adv Med Oncol 2018;10:1758835918794630.

85. Msaouel P, Koutsilieris M. Diagnostic value of circulating tumor cell detection in bladder and urothelial cancer: systematic review and meta-analysis. BMC Cancer 2011;11:336.

86. Retz M, Lehmann J, Roder C, et al. Cytokeratin-20 reverse-transcriptase polymerase chain reaction as a new tool for the detection of circulating tumor cells in peripheral blood and bone marrow of bladder cancer patients. Eur Urol 2001;39:507-15; discussion 516-7.

87. Naoe M, Ogawa Y, Morita J, et al. Detection of circulating urothelial cancer cells in the blood using the CellSearch System. Cancer 2007;109:1439-45.

88. Alva A, Friedlander T, Clark M, et al. Circulating Tumor Cells as Potential Biomarkers in Bladder Cancer. J Urol 2015;194:790-8.

89. Rink M, Chun FK, Minner S, et al. Detection of circulating tumour cells in peripheral blood of patients with advanced non-metastatic bladder cancer. BJU Int 2011;107:1668-75.

90. Okegawa T, Hayashi K, Hara H, et al. Immunomagnetic quantification of circulating tumor cells in patients with urothelial cancer. Int J Urol 2010;17:254-8.

91. Karl A, Tritschler S, Hofmann S, et al. Perioperative search for circulating tumor cells in patients undergoing radical cystectomy for bladder cancer. Eur J Med Res 2009;14:487-90.

92. Lu JJ, Kakehi Y, Takahashi T, et al. Detection of circulating cancer cells by reverse transcription-polymerase chain reaction for uroplakin II in peripheral blood of patients with urothelial cancer. Clin Cancer Res 2000;6:3166-71.

93. Ribal MJ, Mengual L, Marin M, et al. Molecular staging of bladder cancer with RT-PCR assay for CK20 in peripheral blood, bone marrow and lymph nodes: comparison with standard histological staging. Anticancer Res 2006;26:411-9.

94. Guzzo TJ, McNeil BK, Bivalacqua TJ, et al. The presence of circulating tumor cells does not predict extravesical disease in bladder cancer patients prior to radical cystectomy. Urol Oncol 2012;30:44-8.

95. Zhang Z, Fan W, Deng Q, et al. The prognostic and diagnostic value of circulating tumor cells in bladder cancer and upper tract urothelial carcinoma: a metaanalysis of 30 published studies. Oncotarget 2017;8:59527.

96. Contreras-Sanz A, Roberts ME, Seiler R, et al. Recent progress with next-generation biomarkers in muscleinvasive bladder cancer. Int J Urol 2017;24:7-15.

97. Jiang P, Chan KCA, Lo YMD. Liver-derived cell-free nucleic acids in plasma: Biology and applications in liquid biopsies. J Hepatol 2019;71:409-21.

98. Yang Y, Miller CR, Lopez-Beltran A, et al. Liquid Biopsies in the Management of Bladder Cancer: Next-Generation Biomarkers for Diagnosis, Surveillance, and TreatmentResponse Prediction. Crit Rev Oncog 2017;22:389-401.

99. Tan MP, Attard G, Huddart RA. Circulating Tumour DNA in Muscle-Invasive Bladder Cancer. Int J Mol Sci 2018. doi: 10.3390/ijms19092568.

100. Nagy RJ, Agarwal N, Gupta S, et al. Circulating cellfree DNA profiling of patients with advanced urothelial carcinoma of the bladder. J Clin Oncol 2016;34:4528.

101. Grivas P, Lalani AA, Pond GR, et al. Circulating Tumor DNA Alterations in Advanced Urothelial Carcinoma and Association with Clinical Outcomes: A Pilot Study. Eur Urol Oncol 2020;3:695-9.

102. Gootenberg JS, Abudayyeh OO. Nucleic acid detection with CRISPR-Cas13a/C2c2. 2017;356:438-42.

103. Kim YH, Yan C, Lee IS, et al. Value of urinary topoisomerase-IIA cell-free DNA for diagnosis of bladder cancer. Investig Clin Urol 2016;57:106-12.

104. Saliba AE, Westermann AJ, Gorski SA, et al. Single-cell RNA-seq: advances and future challenges. Nucleic Acids Res 2014;42:8845-60.

105. Ono H, Arai Y, Furukawa E, et al. Single-cell DNA and RNA sequencing reveals the dynamics of intra-tumor heterogeneity in a colorectal cancer model. bioRxiv 2019. doi: 10.1101/616870.

106.Zhang X, Zhang M, Hou Y, et al. Single-cell analyses of transcriptional heterogeneity in squamous cell carcinoma of urinary bladder. Oncotarget 2016;7:66069-76.

107.Ellsworth DL, Blackburn HL, Shriver CD, et al. Single-cell sequencing and tumorigenesis: improved 
understanding of tumor evolution and metastasis. Clin Transl Med 2017;6:15.

108. Schmidt F, Efferth T. Tumor Heterogeneity, Single-Cell Sequencing, and Drug Resistance. Pharmaceuticals (Basel) 2016. doi: 10.3390/ph9020033.

Cite this article as: Kang HW, Kim WJ, Choi W, Yun SJ. Tumor heterogeneity in muscle-invasive bladder cancer. Transl Androl Urol 2020;9(6):2866-2880. doi: 10.21037/tau.2020.03.13
109. Tanaka N, Katayama S, Reddy A, et al. Single-cell RNA-seq analysis reveals the platinum resistance gene COX7B and the surrogate marker CD63. Cancer Med 2018;7:6193-204. 